\title{
Quality of care in early detection and management of pre-eclampsia/eclampsia in health facilities in Afghanistan
}

Nasratullah Ansari ${ }^{1,2^{*}}$ D, Partamin Manalai ${ }^{3}$, Farzana Maruf ${ }^{3}$, Sheena Currie ${ }^{1}$, Jelle Stekelenburg ${ }^{4,5}$, Jos van Roosmalen ${ }^{2}$, Young-Mi Kim ${ }^{1}$ and Hannah Tappis ${ }^{1}$

\begin{abstract}
Background: Afghanistan faces a high burden of maternal and neonatal morbidity and mortality. Hypertensive disorders of pregnancy, including pre-eclampsia and eclampsia (PE/E), are among the most common causes of maternal and neonatal complications. Hypertensive disorders of pregnancy can lead to fatal complications for both the mother and fetus. The 2016 Afghanistan National Maternal and Newborn Health Quality of Care Assessment assessed quality of early detection and management of PE/E in health facilities and skilled birth attendants' (SBAs) perceptions of their working environment.

Methods: All accessible public health facilities with an average of at least five births per day $(n=77)$, a nationally representative sample of public health facilities with less than five births per day $(n=149)$, and 20 purposively selected private health facilities were assessed. Methods included a facility inventory and record review, interviews with SBAs, and direct clinical observation of antenatal care (ANC), intrapartum care and immediate postnatal care (PNC), as well as severe PE/E case management.

Results: Most facilities had supplies and medicines for early detection and management of PE/E. At public health facilities, 357 of 414 (86.2\%) clients observed during ANC consultations had their blood pressure checked and 159 (38.4\%) were asked if they had experienced symptoms of PE/E. Only 553 of 734 (72.6\%) SBAs interviewed were able to correctly identify severe pre-eclampsia described in a case scenario. Of 29 PE/E cases observed, 17 women (59\%) received the correct loading dose of magnesium sulfate (MgSO4) and 12 women (41\%) received the correct maintenance dose of $\mathrm{MgSO}_{4}$.

At private health facilities, 39 of 45 ANC clients had their blood pressure checked and 9 of 45 (20\%) were asked about symptoms of PE/E. Fifty-four of 64(84.4\%) SBAs in private facilities correctly identified severe pre-eclampsia described in a case scenario.

Conclusion: Notable gaps in SBAs' knowledge and clinical practices in detection and management of PE/E in various health facilities increase the risk of maternal and perinatal mortality. Continuing education of health care providers and increased investment in focused quality improvement initiatives will be critical to improve the quality of health care services in Afghanistan.
\end{abstract}

Keywords: Pre-eclampsia, eclampsia, maternal health, quality of health care, Afghanistan

\footnotetext{
* Correspondence: nasrat.ansari@jhpiego.org

'Jhpiego, 1615 Thames Street, Baltimore, MD, USA

${ }^{2}$ Athena Institute, Faculty of Science, Vrije Universiteit, Amsterdam, De

Boelelaan 1105, 1081, HV, Amsterdam, the Netherlands

Full list of author information is available at the end of the article
}

(c) The Author(s). 2019 Open Access This article is distributed under the terms of the Creative Commons Attribution 4.0 International License (http://creativecommons.org/licenses/by/4.0/), which permits unrestricted use, distribution, and reproduction in any medium, provided you give appropriate credit to the original author(s) and the source, provide a link to the Creative Commons license, and indicate if changes were made. The Creative Commons Public Domain Dedication waiver (http://creativecommons.org/publicdomain/zero/1.0/) applies to the data made available in this article, unless otherwise stated. 


\section{Background}

Hypertensive disorders of pregnancy, including preeclampsia/eclampsia (PE/E), are the second most common cause of maternal mortality, accounting for $14.1 \%$ of maternal deaths, and are associated with fetal and neonatal mortality worldwide [1]. PE/E complicates $3-5 \%$ of pregnancies and occurs in the second half of pregnancy, during labor, and in the postpartum period [2]. It is one of the most common causes of preterm birth [3].

Afghanistan has one of the highest burdens of maternal mortality in the world, estimated at 789 deaths per 100,000 live births [4]. The 2010 Afghanistan Mortality Survey showed that hypertensive disorders of pregnancy account for $20 \%$ of maternal deaths, and are the second most common cause of death after obstetric hemorrhage [5]. Although the 2015 Afghanistan Demographic Health Survey did not examine causes of maternal mortality, a 2011 study showed that hypertensive disorders of pregnancy are the leading cause of maternal mortality in two districts reflective of the most urban (Kabul City) and most remote (Ragh district, Badakshan) areas in Afghanistan [6].

Administration of magnesium sulfate $(\mathrm{MgSO} 4)$ and timely childbirth can prevent most maternal deaths due to PE/E. Ensuring timely and effective care requires appropriate use of evidence-based clinical and nonclinical interventions, strengthened health infrastructure, and motivated and competent health care providers $[7,8]$.

A 2010 study showed that supplies for prevention and treatment of $\mathrm{PE} / \mathrm{E}$ were available in most health facilities in Afghanistan and providers were relatively well-prepared to manage severe PE/E cases [9]. Since 2010, interventions to improve quality and coverage of maternal and newborn health $(\mathrm{MNH})$ care have been implemented, but the security situation in Afghanistan has deteriorated [10], resulting in facility closure and increased staff turnover [11]. Impacts on facility readiness and quality detection and management of PE/E at different levels of public health facilities are not known, and no study to date has examined readiness or quality of detection and management of PE/E at private health facilities.

This study examines the quality of early detection and management of $\mathrm{PE} / \mathrm{E}$ in public and private health facilities in 2016, documents SBAs' perceptions of their working environment, and assesses whether quality of care varies by different levels of public health facilities. Such information is crucial to identify strengths and weaknesses in the quality of care in public and private health facilities, to help to frame policy discussions, and to design evidence-based interventions to address these challenges in the country.

\section{Methods}

\section{Study design}

The 2016 Afghanistan Maternal and Newborn Health Quality of Care Assessment was a cross-sectional assessment examining facility readiness and quality of routine $\mathrm{MNH}$ services and management of obstetric and newborn complications. This study uses the subset of data on early detection and management of PE/E.

The 2016 Afghanistan Maternal and Newborn Health Quality of Care Assessment included three data collection methods: a facility inventory and record-review tool to verify availability of medications, supplies, and equipment, as well as human resources, infrastructure, and facility records; an interview tool to collect information on SBAs' knowledge, practices, and attitudes, and constraints faced with the provision of antenatal care (ANC), labor/birth, and postnatal care (PNC) services; and direct clinical observation checklists to document ANC, labor/birth, inpatient PNC before discharge, and management of selected obstetric and newborn complications, including severe PE/E.

Observation checklist content was based on World Health Organization (WHO) guidelines and adapted from tools used in conducting quality of care assessments in other countries [12]; the content of other tools was adapted from Demographic and Health Survey Service Provision Assessment [13], and emergency obstetric and newborn care (EmONC) assessments supported by the Averting Maternal Death and Disability (AMDD) program [14]. All tools were developed in English and translated into Dari and Pashto.

\section{Sample}

Ministry of Public Health (MoPH) guidelines require specialized hospitals (SHs), regional hospitals (RHs) provincial hospitals (PHs) and district hospitals (DHs) to provide all comprehensive EmONC signal functions [15]; and primary health care facilities including as comprehensive health centers $(\mathrm{CHCs})$, basic health centers (BHCs), sub-health centers (SHCs) and family health houses $[\mathrm{FHH}]$ to provide all basic EmONC signal functions, including early detection and management of $\mathrm{PE} /$ $\mathrm{E}$ and refer women with complications to higher levels of care when needed [16, 17].

The 2016 Afghanistan Maternal and Newborn Health Quality of Care Assessment was designed to assess readiness and quality of care at a census of all public facilities with an average of five or more deliveries per day, and to assess readiness at a nationally representative sample of public facilities with an average of zero to four deliveries per day (Fig. 1). Facility caseloads were determined based on data reported in the national health management information system: 79 public health facilities reported an average of at least five deliveries 


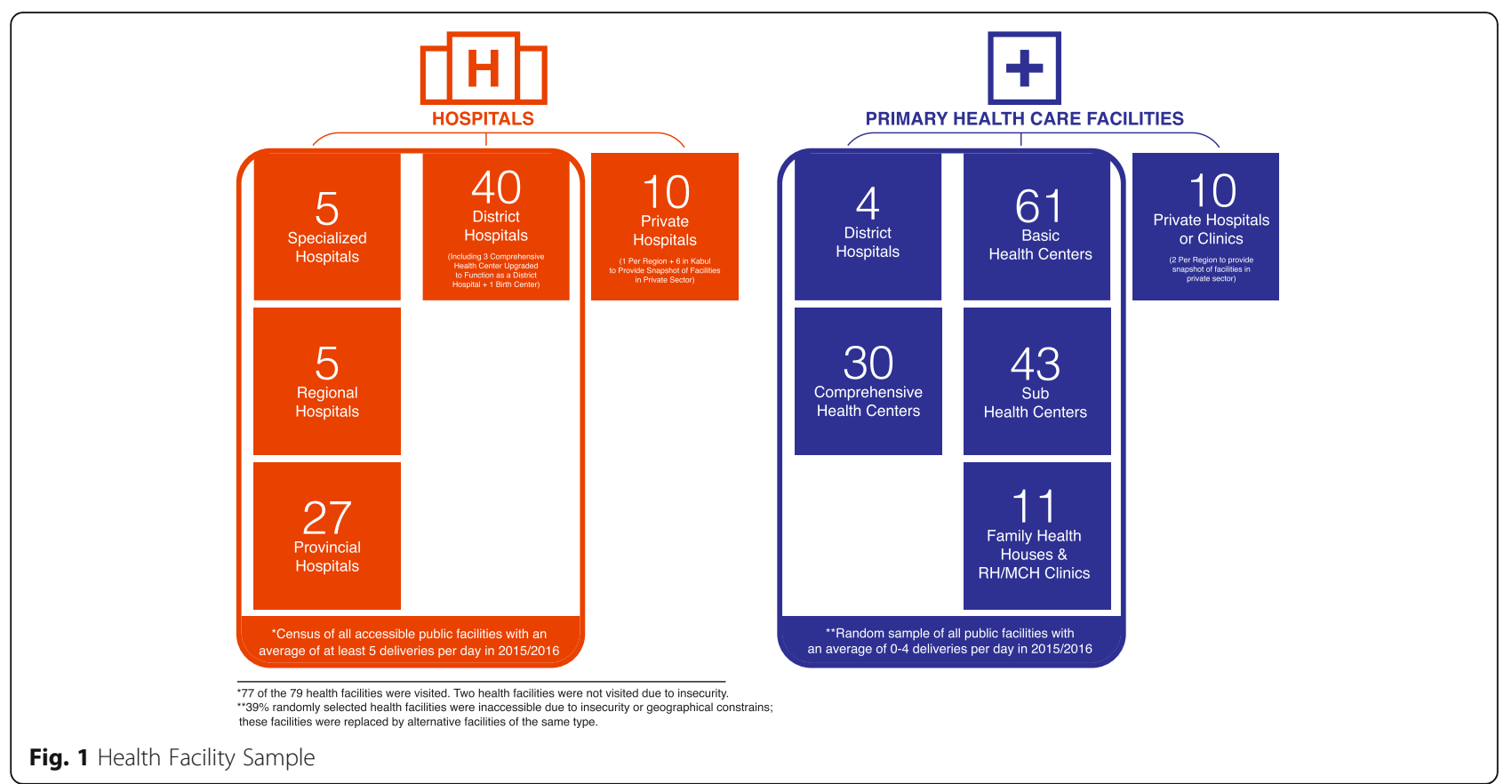

per day, 386 reported an average of 1-4 deliveries per day, and 1351 did not report any deliveries in the year prior to the assessment.

To obtain a nationally representative sample of public facilities with fewer than five births per day, we used probability proportional to size methods of cluster sampling to estimate results by facility type. Using a design effect of $1.5 \%$, we estimated that a total of 147 public health facilities would need to be assessed to estimate results with $+10 \%$ precision, $95 \%$ confidence: four DHs, 30 CHCs, 61 BHCs, 43 SHCs, and 11 FHHs.

Twenty private sector facilities (10 hospitals with at least five deliveries per day, and 10 health centers with fewer than five deliveries per day) were purposively sampled to provide a snapshot of private facilities in all regions of the country. This sample is not statistically representative for all private sector facilities. The private health sector sampling is not comparable to public health sector sampling.

\section{Data collection, sites, and procedure}

Data collectors were 32 experienced female SBAs (midwives and doctors) who successfully completed a May 2016 training on conducting clinical observations. Training sessions, which were held in Kabul, consisted of technical updates on maternal and newborn care (routine and selected complications), clinical site data collection techniques with a focus on clinical observation as well as data quality assurance, research ethics, and using CommCare software for data collection.

Data collection at health facilities with an average of at least five deliveries per day (including facility inventory/ record review, SBA interviews, and direct observations of clinical care) was completed between May 14, 2016 and August 3, 2016. Data collection at health facilities that averaged fewer than five deliveries per day (facility inventory/record review and SBA interviews only) was completed from November 5, 2016 to January 5, 2017.

Data collection teams visited 77 of the 79 public health facilities with an average of at least five deliveries per day. Data collectors were not able to visit two health facilities due to insecurity surrounding them. Each team consisted of three data collectors and aimed to complete data collection within two to three days per facility. Due to insecurity or geographical and climatic constraints, $39 \%$ of randomly selected public health facilities with zero to four deliveries per day were inaccessible; therefore, these facilities were replaced by alternative facilities of the same type, following standardized replacement sampling protocols.

Interviews were conducted with 333 health providers at SHs, RHs, and PHs; 228 at high volume DHs with more than five deliveries per day; 69 at low volume DHs and $\mathrm{CHCs} ; 104$ at BHCs, SHCs, and FHHs; and 64 at private health facilities. Four hundred and fourteen ANC observations were conducted at public health facilities and 45 were completed at private health facilities; 507 childbirth observations took place at public health facilities and 31 at private health facilities; and 402 PNC observations were conducted at public health facilities and 30 at private health facilities.

Data collection teams in both phases used CommCare software on Android tablet computers. Where use of the tablets was considered a security risk (for example, 
where there was a risk of robbery), data collectors used paper tools, but loaded the contents of these tools to the tablets when they were in a safe location with Internet access. Logic, skip, and consistency checks were built into the program, and data collectors were trained to review records for missing or inconsistent answers before submission.

\section{Data analysis}

For analysis purposes, facility types were defined as follows: 1) specialized, regional, and provincial hospitals; 2) district hospitals with an average of five or more deliveries per day; 3 ) district hospitals and comprehensive health centers with an average of less than five deliveries per day; and 4) basic health centers, sub-health centers, family health houses, and other primary health care facilities. These categories ensure that indicators based on direct observation of clinical services can be estimated with $+10 \%$ precision, and that facilities with similar management mechanisms and service packages are grouped together.

Descriptive statistics were used for analysis. A chi-squared test was used to test for differences in facility readiness and routine care practices by public facility type. All statistical analyses were conducted using Stata ${ }^{\circ}$. Data from private facilities are based on purposive nonrandom sampling and are not intended for direct comparison compared with the public health facility random sampling data.

PE/E case management data analysis focused on four key areas in only public health facilities: blood pressure (BP) and proteinuria testing and anticonvulsant and antihypertensive therapy. In both severe pre-eclampsia and eclampsia, BP check, proteinuria tests and administration of antihypertensive data was analyzed. For anticonvulsant therapy, correct loading and maintenance doses of $\mathrm{MgSO} 4$ were assessed based on national case management guidelines aligned with WHO Managing Complications in Pregnancy and Childbirth (MCPC) 2007 guidelines [18].

\section{Ethical considerations}

The ethical review boards of the Afghanistan MoPH and John Hopkins Bloomberg School of Public Health (JHSPH) in Baltimore, Maryland approved the 2016 National Maternal and Newborn Health Quality of Care Assessment protocol (MoPH IRB \#36153, JHSPH IRB \#6799). Written permission for data collection was obtained from facility directors, and oral informed consent was obtained from all participating health care providers and clients (or a client's next of kin if the woman was too ill to provide informed consent directly).

\section{Results}

Characteristics of health facilities and health care providers In 144 of 226 public health facilities (63.7\%), facility management reported providing 24-h services, but availability of $24-\mathrm{h}$ care was higher $(100 \%)$ in $\mathrm{SH} / \mathrm{RH} / \mathrm{PHs}$ and high volume DHs, and lower (56.8\%) in low volume $\mathrm{DH} / \mathrm{CHC}$ and $(41.2 \%)$ in BHC/SHC/FHH $(p<0.001)$. Of 734 SBAs interviewed, 173 (23.6\%) had received training in BEmONC in the last 3 years, and $186(25.3 \%)$ had received training specifically in management of $\mathrm{PE} / \mathrm{E}$. (Table 1).

\section{Availability of supplies, equipment, and drugs}

Of 226 public health facilities, 178 (78.8\%) had injectable $\mathrm{MgSO} 4$. The availability of MgSO4 did not differ significantly between facility types $(p=0.370)$. Injectable calcium gluconate, the antidote for $\mathrm{MgSO} 4$, was available in 113 of $226(50.0 \%)$ health facilities, including more than $80 \%$ of $\mathrm{SHs} / \mathrm{RHs} / \mathrm{PHs}$ and high volume DHs, and less than 35\% low volume DHs/ CHCs and BHCs/SHCs/ FHHs $(p<0.001)$ (Table 2).

The majority of public health facilities assessed had injectable oxytocin in the delivery room (199 of 226; $88.1 \%$ ); misoprostol was available in over $50 \%$ of $\mathrm{PH} /$ $\mathrm{RH} / \mathrm{SH}$ and high volume $\mathrm{DH}$, and less than $14 \%$ of low volume $\mathrm{DH} / \mathrm{CHC}$ and $\mathrm{BHC} / \mathrm{SHC} / \mathrm{FHH}(p<0.001)$ (Table 2).

Of 226 public health facilities, 194 (85.8\%) had functioning BP machines, 199 (88.1\%) had functioning stethoscopes, and $194(85.8 \%)$ had functioning fetoscopes. (Table 2). Less than half of the health facilities (102 of 226; 45.1\%) had EmONC guidelines and protocols available in the delivery rooms (Table 2).

\section{SBA knowledge on early detection and management of $\mathrm{PE} / \mathrm{E}$}

SBAs were given descriptions of cases with obstetric complications and asked to provide a diagnosis. Overall, 553 of 734 (72.6\%) SBAs correctly identified a case with severe headache, blurred vision, BP 160/120, and 3+ proteinuria as severe pre-eclampsia (Table 3 ).

Of 734 SBAs interviewed, 606 (82.6\%) knew that $\mathrm{MgSO} 4$ was essential for management of severe pre-eclampsia. SBAs' knowledge of $\mathrm{MgSO} 4$ varied significantly by health facility type, ranging from 272 of 333 (81.7\%) in $\mathrm{PH} / \mathrm{RH} / \mathrm{SH}$ and 176 of 228 (77.2\%) high volume DHs to 66 of 69 (95.7\%) in low volume DH/CHC and 92 of $104(88.5 \%)$ in $\mathrm{BHC} / \mathrm{SHC} / \mathrm{FHH}(p=0.001)$. In addition, 318 of 734 (43.3\%) SBAs knew that preparing women for childbirth within $24 \mathrm{~h}$ was an essential action in the management of severe pre-eclampsia, with over $56 \%$ of SBAs from $\mathrm{PH} / \mathrm{RH} / \mathrm{SH}$ and high volume $\mathrm{DH}$ and less than $30 \%$ of SBAs from low volume $\mathrm{DH} / \mathrm{CHC}$ and $\mathrm{BHC} / \mathrm{SHC} / \mathrm{FHH}$ demonstrating this 


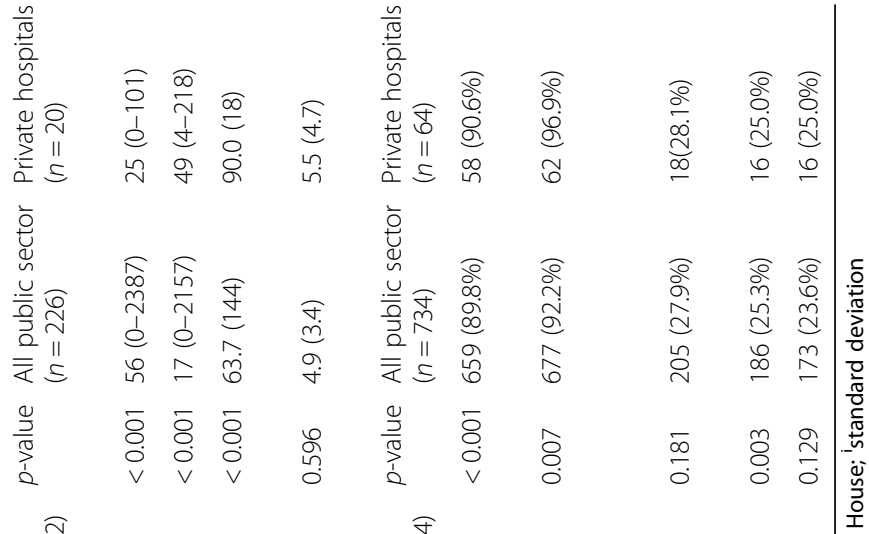

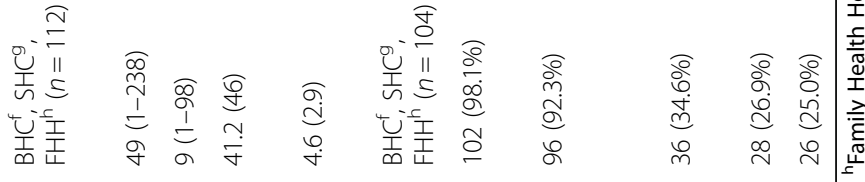

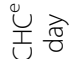

등

+

妾 $\frac{5}{\sqrt{0}}$

돔

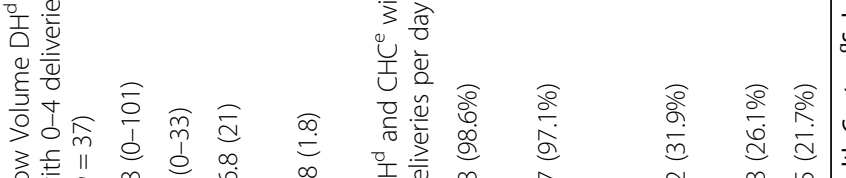

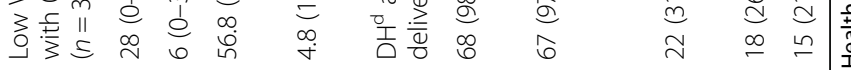

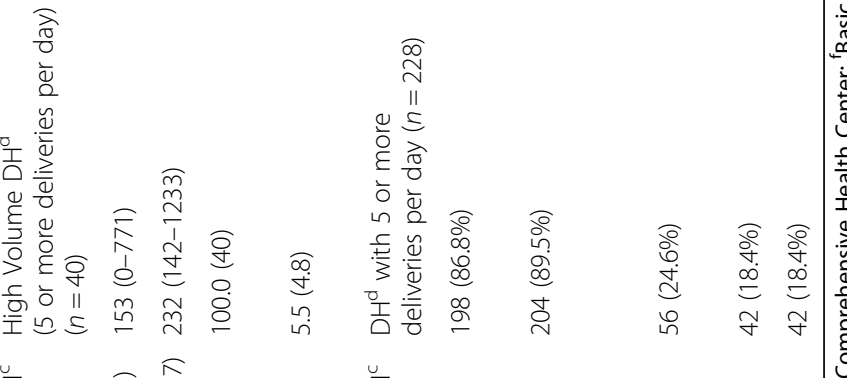

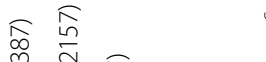

ग व

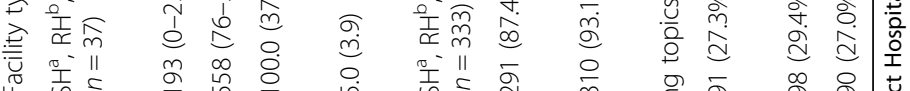

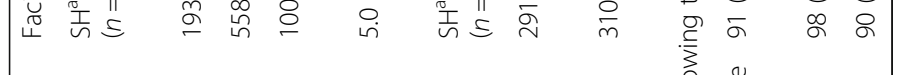


Table 2 Supply, equipment and drug for early detection and management of pre-eclampsia/eclampsia (PE/E) available at the point of care

\begin{tabular}{|c|c|c|c|c|c|c|c|}
\hline \multirow[t]{2}{*}{ Available items number (\%) } & \multicolumn{7}{|l|}{ Facility type } \\
\hline & $\begin{array}{l}\overline{\mathrm{SH}^{\mathrm{a}}, \mathrm{RH}^{\mathrm{b}}, \mathrm{PH}^{\mathrm{c}}} \\
(n=37)\end{array}$ & $\begin{array}{l}\text { High Volume } \mathrm{DH}^{\mathrm{d}} \\
\text { (5 or more deliveries } \\
\text { per day) }(n=40)\end{array}$ & $\begin{array}{l}\text { Low Volume } \mathrm{DH}^{\mathrm{d}} \text { and } \\
\mathrm{CHC}^{\mathrm{e}}(0-4 \text { deliveries } \\
\text { per day })(n=37)\end{array}$ & $\begin{array}{l}\mathrm{BHC}^{\mathrm{f}}, \mathrm{SHC}^{\mathrm{g}}, \\
\mathrm{h}_{\mathrm{FHH}} \\
(n=112)\end{array}$ & $p$-value & $\begin{array}{l}\text { All public } \\
\text { sector } \\
(n=226)\end{array}$ & $\begin{array}{l}\text { Private } \\
\text { hospitals } \\
(n=20)\end{array}$ \\
\hline $\begin{array}{l}\text { Guidelines or national treatment } \\
\text { protocol for emergency obstetric } \\
\text { and newborn care }\end{array}$ & $16(43.2 \%)$ & $19(47.5 \%)$ & $20(54.1 \%)$ & $47(42.0 \%)$ & 0.527 & $102(45.1 \%)$ & $7(35.0 \%)$ \\
\hline Functioning blood pressure apparatusi & $30(81.1 \%)$ & $34(85.0 \%)$ & $35(94.6 \%)$ & $95(84.8 \%)$ & 0.303 & $194(85.8 \%)$ & $15(75.0 \%)$ \\
\hline Functioning stethoscope & $29(78.4 \%)$ & $36(90.0 \%)$ & $35(94.6 \%)$ & 99 (88.4\%) & 0.702 & 199 (88.1\%) & $17(85.0 \%)$ \\
\hline $\begin{array}{l}\text { Functioning fetal stethoscope } \\
\text { or fetoscope }\end{array}$ & $25(67.6 \%)$ & $36(90.0 \%)$ & $34(91.9 \%)$ & 99 (88.4\%) & 0.094 & $194(85.8 \%)$ & $15(75.0 \%)$ \\
\hline Injectable oxytocin & $33(89.2 \%)$ & $36(90.0 \%)$ & $36(97.3 \%)$ & $94(83.9 \%)$ & 0.637 & 199 (88.1\%) & $18(90.0 \%)$ \\
\hline Misoprostol & $21(56.8 \%)$ & $21(52.5 \%)$ & $5(13.5 \%)$ & $10(8.9 \%)$ & $<0.001$ & $57(25.2 \%)$ & $12(60.0 \%)$ \\
\hline Injectable diazepam & $28(75.7 \%)$ & $29(72.5 \%)$ & $25(67.6 \%)$ & $49(43.8 \%)$ & $<0.001$ & $131(58.0 \%)$ & $11(55.0 \%)$ \\
\hline Injectable magnesium sulfate & $33(89.2 \%)$ & 36 (90.0\%) & $28(75.7 \%)$ & $81(72.3 \%)$ & 0.370 & $178(78.8 \%)$ & $18(90.0 \%)$ \\
\hline Injectable calcium gluconate & $31(83.8 \%)$ & $32(80.0 \%)$ & $11(29.7 \%)$ & 39 (34.82\%) & $<0.001$ & $113(50.0 \%)$ & $15(75.0 \%)$ \\
\hline
\end{tabular}

${ }^{\mathrm{a}}$ Specialty Hospital; ${ }^{\mathrm{b}}$ Regional Hospital; ${ }^{\mathrm{C}}$ Provincial Hospital; ${ }^{\mathrm{d}}$ District Hospital; ${ }^{\mathrm{e}}$ Comprehensive Health Center; ${ }^{\mathrm{f}}$ Basic Health Center; ${ }^{\mathrm{g}}$ Sub-Health Center; ${ }^{\mathrm{h}}$ Family Health House; 'blood pressure apparatus includes any type of sphyngomanometer

knowledge $(p<0.001)$. Moreover, 417 of $734(56.8 \%)$ SBAs knew that antihypertensive medication was essential for management of severe pre-eclampsia, with more than $57 \%$ of SBAs from $\mathrm{PH} / \mathrm{RH} / \mathrm{SH}$ and high volume $\mathrm{DH}$ and less than $48 \%$ of SBAs from low volume $\mathrm{DH} / \mathrm{CHC}$ and $\mathrm{BHC} / \mathrm{SHC} / \mathrm{FHH}$ answering questions about this correctly $(p<0.001)$ (Table 3$)$.

\section{Direct observation during ANC, labor/birth, and inpatient} PNC for early detection of PE/E

SBAs in public health facilities measured BP of 357 of 414 (86.2\%) women observed during ANC, 320 of 507 (63.1\%) women observed during the first and second stage of labor, and 290 of $402(72.1 \%)$ women observed in the postnatal ward.

Table 3 Skilled birth attendant (SBA) knowledge on early detection and management of pre-eclampsia/eclampsia (PE/E)

\begin{tabular}{|c|c|c|c|c|c|c|c|}
\hline \multirow{2}{*}{$\begin{array}{l}\text { SBA demonstrating knowledge of PE/E } \\
\text { early detection and management } \\
\text { number }(\%)\end{array}$} & \multicolumn{7}{|l|}{ Facility type } \\
\hline & $\begin{array}{l}\mathrm{SH}^{\mathrm{a}}, \mathrm{RH}^{\mathrm{b}}, \mathrm{PH}^{\mathrm{c}} \\
(n=333)\end{array}$ & $\begin{array}{l}\text { High Volume } \mathrm{DH}^{\mathrm{d}} \\
\text { (5 or more deliveries } \\
\text { per day) }(n=228)\end{array}$ & $\begin{array}{l}\text { Low Volume } \mathrm{DH}^{\mathrm{d}} \text { and } \\
\mathrm{CHC}^{\mathrm{e}}(0-4 \text { deliveries } \\
\text { per day }(n=69)\end{array}$ & $\begin{array}{l}\mathrm{BHC}^{\mathrm{f}}, \mathrm{SHC}^{\mathrm{g}}, \\
\mathrm{FHH}^{\mathrm{h}} \\
(n=104)\end{array}$ & $p$-value & $\begin{array}{l}\text { All public } \\
\text { sector } \\
n=734)\end{array}$ & $\begin{array}{l}\text { Private } \\
\text { hospitals } \\
(n=64) \\
\end{array}$ \\
\hline \multicolumn{8}{|c|}{ Know essential actions to take in management of severe pre-eclampsia at term } \\
\hline Administer magnesium sulfate & $272(81.7 \%)$ & $176(77.2 \%)$ & $66(95.7 \%)$ & $92(88.5 \%)$ & $<0.001$ & $606(82.6 \%)$ & $56(87.5 \%)$ \\
\hline Administer antihypertensives & $205(61.6 \%)$ & $132(57.9 \%)$ & $33(47.8 \%)$ & $47(45.2 \%)$ & $<0.001$ & $417(56.8 \%)$ & $34(53.1 \%)$ \\
\hline Prepare to deliver within $24 \mathrm{~h}$ & $167(50.2 \%)$ & $106(46.5 \%)$ & $14(20.3 \%)$ & $31(29.8 \%)$ & $<0.001$ & $318(43.3 \%)$ & $27(42.2 \%)$ \\
\hline $\begin{array}{l}\text { Incorrectly responded and listed } \\
\text { administering diazepam as } \\
\text { appropriate management of severe } \\
\text { pre-eclampsia at term }\end{array}$ & $99(29.7 \%)$ & $63(27.6 \%)$ & $44(63.8 \%)$ & $56(53.9 \%)$ & $<0.001$ & $262(35.7 \%)$ & $25(39.1 \%)$ \\
\hline $\begin{array}{l}\text { Know to check maternal blood pressure } \\
\text { during postnatal care }\end{array}$ & $241(72.4 \%)$ & $170(74.6 \%)$ & $61(88.4 \%)$ & $90(86.5 \%)$ & $<0.001$ & $562(76.6 \%)$ & $52(81.3 \%)$ \\
\hline $\begin{array}{l}\text { Know correct diagnosis of severe } \\
\text { pre-eclampsia from a case description }\end{array}$ & $224(67.3 \%)$ & $158(69.3 \%)$ & $60(87.0 \%)$ & $91(87.5 \%)$ & 0.001 & $553(72.6 \%)$ & $54(84.4 \%)$ \\
\hline \multicolumn{8}{|c|}{ Know the following procedures that are carried out routinely for all patients during labor and delivery } \\
\hline $\begin{array}{l}\text { Know to monitor maternal blood } \\
\text { pressure }\end{array}$ & $258(77.5 \%)$ & $170(74.6 \%)$ & 66 (95.7\%) & 92 (88.5\%) & $<0.001$ & $586(79.8 \%)$ & $56(87.5 \%)$ \\
\hline $\begin{array}{l}\text { Know to monitor intermittent fetal } \\
\text { heart rate (approx. every hour or } \\
\text { more often) }\end{array}$ & $236(70.9 \%)$ & $170(74.6 \%)$ & 65 (94.2\%) & 90 (86.5\%) & $<0.001$ & 561 (76.4\%) & 52 (81.3\%) \\
\hline
\end{tabular}

${ }^{\mathrm{a}}$ Specialty Hospital; ${ }^{\mathrm{b}}$ Regional Hospital; ${ }^{\mathrm{C}}$ Provincial Hospital; ${ }^{\mathrm{d} D i s t r i c t}$ Hospital; ${ }^{\mathrm{e}}$ Comprehensive Health Center; ${ }^{\mathrm{f} B a s i c}$ Health Center; ${ }^{9}$ Sub-Health Center; ${ }^{\text {h}}$ Family Health House; 
SBAs asked if women had recently or were currently experiencing severe headache or blurred vision in 159 of 414 (38.4\%) ANC consultations observed, 59 of 436 (13.5\%) women observed during childbirth and 124 of $402(30.8 \%)$ women observed in the postnatal ward.

SBAs counseled women on the need for care seeking if experiencing convulsions and loss of consciousness in 108 of 414 (26.1\%) ANC consultations and 115 of 402 (28.6\%) inpatient PNC consultations before discharge after childbirth. Similarly, they provided counseling on the need for care seeking if experiencing severe headache with blurred vision in 150 of 414 (36.2\%) of ANC consultations and 143 of 402 (35.6\%) of PNC consultations (Table 4).

\section{Direct observation of severe PE/E case management}

Data collectors documented management of 33 cases of $\mathrm{PE} / \mathrm{E}$ during visits to public health facilities with an average of at least five births per day. These 33 observations took place at 22 different health facilities; 26 were observed at $\mathrm{SH} / \mathrm{RH} / \mathrm{PHs}$ and seven at high volume DHs. Two women's conditions were classified as mild pre-eclampsia and another two cases had insufficient data for classification. These four women were excluded from analysis. Of the 29 remaining PE/E cases, 18 women's conditions were identified as severe pre-eclampsia and 11 as eclampsia (Table 5).

Twenty-eight of 29 women with severe PE/E had their BP measured. In one woman, the assessor did not observe BP measurement and could not ascertain from the woman's records whether her BP was checked.

Of 29 women with severe PE/E, 17 (59\%) received the correct $\mathrm{MgSO} 4$ loading dose (Table 5). Four women had insufficient data recorded to determine if the correct dose was administered. Of 29 women with severe PE/E, 12 women (41\%) received the correct maintenance dose of MgSO4. Six cases had insufficient data recorded to determine correct maintenance dose.

The PE/E case management observation checklist documented whether antihypertensives were administered but did not collect information on dosage. Of 29 women with severe PE/E, 20 (69\%) received and eight (28\%) did not receive any type of antihypertensive drug. In one case of eclampsia, it is unknown if the woman received antihypertensives (Table 5).

\section{SBA perceptions of working conditions}

Of 734 SBAs interviewed at public health facilities, 497 $(67.7 \%)$ reported feeling that their supervisors treat them with respect. More than half of SBAs interviewed (434 of $734 ; 59.1 \%)$ mentioned the need for more knowledge, including approximately $60 \%$ of SBAs at $\mathrm{PH} / \mathrm{RH} / \mathrm{SH}$ and high volume $\mathrm{DH}$ and over $72 \%$ of respondents from low volume $\mathrm{DH} / \mathrm{CHC}$ and $\mathrm{BHC} / \mathrm{SHC} / \mathrm{FHH} \quad(p<0.001)$. One-third of respondents (249 of 734; 33.9\%) mentioned good security in the workplace as an important factor in ensuring an enabling environment for quality care provision, including more than $42 \%$ of SBAs from high volume DHs and almost 34\% of SBAs from all other facility types indicated this need $(p=0.009)$ (Table 6).

Almost a quarter of SBAs (168 of $734 ; 22.9 \%)$ expressed concerns about safety while traveling to public health facilities. Lack of transport was another challenge emphasized by 152 of 734 (20.7\%), including almost $20 \%$ of SBAs working in $\mathrm{PH} / \mathrm{RH} / \mathrm{SH}$ and high volume $\mathrm{DH}$,

Table 4 Early detection of pre-eclampsia/eclampsia (PE/E) during antenatal care (ANC), childbirth and postnatal care (PNC) observed on assessment day

\begin{tabular}{|c|c|c|c|c|c|c|}
\hline \multirow{3}{*}{$\begin{array}{l}\text { Cases with key PE/E practices observed } \\
\text { number \# (\%) }\end{array}$} & \multicolumn{6}{|l|}{ Facility type } \\
\hline & \multicolumn{2}{|l|}{ Antenatal care } & \multicolumn{2}{|l|}{ Childbirth } & \multicolumn{2}{|l|}{ Childbirth } \\
\hline & Public & Private & Public & Private & Public & Private \\
\hline Blood pressure measurement & $357 / 414(86.2 \%)$ & $39 / 45(86.7 \%)$ & $320 / 507(63.1 \%)$ & 27/31 (87.1\%) & $290 / 402(72.1 \%)$ & $23 / 30(76.7 \%)$ \\
\hline Fetal heart rate check (at least once) & - & - & 299/507 (59.0\%) & 25/31 (80.7\%) & N/A & N/A \\
\hline \multicolumn{7}{|l|}{ Assessment for danger signs } \\
\hline Severe headache/blurred vision & 159/414 (38.4\%) & $9 / 45(20.0 \%)$ & $59 / 436(13.5 \%)$ & $7 / 24(29.2 \%)$ & $124 / 402(30.8 \%)$ & $5 / 30(16.7 \%)$ \\
\hline Convulsion/loss consciousness & $87 / 414(21.0 \%)$ & $1 / 45(2.2)$ & $24 / 436(5.5 \%)$ & $1 / 24(4.2 \%)$ & $80 / 402(19.9 \%)$ & $5 / 30(16.7 \%)$ \\
\hline Difficulty in breathing & $117 / 414(28.3 \%)$ & 2/ $45(4.4 \%)$ & 43/436 (9.9\%) & 0/24 (0.0\%) & $72 / 402(17.9 \%)$ & $3 / 30(10.0 \%)$ \\
\hline \multicolumn{7}{|l|}{ Counseling on PE/E danger signs and care seeking } \\
\hline Convulsions or loss of consciousness & 108/414 (26.1\%) & $3 / 45(6.7 \%)$ & - & - & 115/402 (28.6) & $6 / 30(20 \%)$ \\
\hline Severe headache with blurred vision & $150 / 414(36.2 \%)$ & $3 / 45(6.7 \%)$ & - & - & $143 / 402(35.6 \%)$ & $6 / 30(20 \%)$ \\
\hline Fast or difficult breathing & $107 / 414(25.9 \%)$ & $0 / 45(0.0 \%)$ & - & - & $76 / 402(18.9 \%)$ & $5 / 30(16.7 \%)$ \\
\hline When to return for next visit & $278 / 414(67.2 \%)$ & $22 / 45(48.9 \%)$ & - & - & - & - \\
\hline Importance of at least having four ANC visits & $208 / 414(50.2 \%)$ & $10 / 45(22.4 \%)$ & N/A & N/A & N/A & N/A \\
\hline
\end{tabular}


Table 5 Pre-eclampsia/eclampsia case management observed on assessment day

\begin{tabular}{|c|c|c|c|}
\hline Number & $\begin{array}{l}\text { Severe pre-eclampsia } \\
n=18\end{array}$ & $\begin{array}{l}\text { Eclampsia } \\
n=11\end{array}$ & $\begin{array}{l}\text { Total } \\
n=29\end{array}$ \\
\hline \multicolumn{4}{|l|}{ Checking blood pressure (BP) } \\
\hline Blood pressure checked & 18 & 10 & 28 \\
\hline Unknown & 0 & 1 & 1 \\
\hline \multicolumn{4}{|l|}{ Checking proteinuria } \\
\hline Proteinuria checked & 17 & 9 & 26 \\
\hline Unknown & 1 & 2 & 3 \\
\hline \multicolumn{4}{|l|}{ Anticonvulsant therapy } \\
\hline \multicolumn{4}{|l|}{ Administration of magnesium sulfate (MgSO4) loading dose } \\
\hline MgSO4 loading dose administered correctly & 10 & 7 & 17 \\
\hline MgSO4 loading dose administered incorrectly & 5 & 3 & 8 \\
\hline Received a loading dose MgSO4 without indication & - & - & - \\
\hline Insufficient data to determine if loading dose was administered correctly & 3 & 1 & 4 \\
\hline \multicolumn{4}{|l|}{ Administration of magnesium sulfate (MgSO4) maintenance dose } \\
\hline MgSO4 maintenance dose administered correctly & 7 & 5 & 12 \\
\hline MgSO4 maintenance dose administered incorrectly & 7 & 4 & 11 \\
\hline Insufficient data to determine if maintenance dose was administered correctly & 4 & 2 & 6 \\
\hline \multicolumn{4}{|l|}{ Antihypertensive therapy } \\
\hline \multicolumn{4}{|l|}{ Administration of antihypertensive drugs } \\
\hline Antihypertensive drug(s) administered & 13 & 7 & 20 \\
\hline Antihypertensive drug(s) not administered & 5 & 3 & 8 \\
\hline Unknown & 0 & 1 & 1 \\
\hline
\end{tabular}

Table 6 Skilled birth attendants' (SBA) perception on enabling environment

\begin{tabular}{|c|c|c|c|c|c|c|c|}
\hline $\begin{array}{l}\text { SBAs' perception on factor for } \\
\text { improving their work environment } \\
\text { number (\%) }\end{array}$ & $\begin{array}{l}\mathrm{SH}^{\mathrm{a}} / \mathrm{RH}^{\mathrm{b}} / \mathrm{PH}^{\mathrm{c}} \\
(n=333)\end{array}$ & $\begin{array}{l}\text { High Volume } \mathrm{DH}^{\mathrm{d}} \\
(5 \text { or more deliveries } \\
\text { per day) }(n=228)\end{array}$ & $\begin{array}{l}\text { Low Volume } \mathrm{DH}^{\mathrm{d}} \text { and } \\
\mathrm{CHC}^{\mathrm{e}}(0-4 \text { deliveries } \\
\text { per day })(n=69)\end{array}$ & $\begin{array}{l}\mathrm{BHC}^{\mathrm{f}}, \mathrm{SHC}^{\mathrm{g}}, \mathrm{FHH}^{\mathrm{h}} \\
(n=104)\end{array}$ & $p$-value & $\begin{array}{l}\text { All public } \\
\text { sector } \\
(n=734)\end{array}$ & $\begin{array}{l}\text { Private } \\
\text { hospitals } \\
(n=64)\end{array}$ \\
\hline $\begin{array}{l}\text { SBAs who thought that their } \\
\text { supervisors treat them respectfully }\end{array}$ & $218(65.5 \%)$ & $144(63.2 \%)$ & $51(73.9 \%)$ & $84(80.8 \%)$ & 0.014 & $497(67.7 \%)$ & $45(70.3 \%)$ \\
\hline \multicolumn{8}{|c|}{ SBAs identifying the most important factors for improving their work environment to provide good quality health care services } \\
\hline $\begin{array}{l}\text { More knowledge/updates/ } \\
\text { training }\end{array}$ & $197(59.2 \%)$ & $108(47.4 \%)$ & $50(72.5 \%)$ & $79(76.0 \%)$ & $<0.001$ & $434(59.1 \%)$ & $47(73.4 \%)$ \\
\hline $\begin{array}{l}\text { Good quality equipment/ } \\
\text { supplies }\end{array}$ & $94(28.2 \%)$ & $68(29.8 \%)$ & $32(46.4 \%)$ & $33(31.7 \%)$ & 0.158 & $227(30.9 \%)$ & $11(17.2 \%)$ \\
\hline Good security situation & $100(30.0 \%)$ & $97(42.5 \%)$ & $23(33.3 \%)$ & $29(27.9 \%)$ & 0.009 & $249(33.9 \%)$ & $10(15.6 \%)$ \\
\hline More support from supervisor & $100(30.0 \%)$ & $84(36.8 \%)$ & $23(33.3 \%)$ & $34(32.7 \%)$ & 0.414 & $241(32.8 \%)$ & $18(28.1 \%)$ \\
\hline \multicolumn{8}{|c|}{ SBAs noting aspects of life outside work that would affect their ability to perform their jobs } \\
\hline Childcare responsibilities & $79(23.7 \%)$ & $43(18.9 \%)$ & $8(11.6 \%)$ & 17 (16.4\%) & 0.073 & $147(20.0 \%)$ & $2(3.1 \%)$ \\
\hline Household responsibilities & $78(23.4 \%)$ & $56(24.6 \%)$ & $6(8.7 \%)$ & $10(9.6 \%)$ & 0.001 & $150(20.4 \%)$ & $5(7.8 \%)$ \\
\hline Safety in getting to/from work & $63(18.9 \%)$ & $60(26.3 \%)$ & $16(23.2 \%)$ & $29(27.9 \%)$ & 0.113 & $168(22.9 \%)$ & $9(14.1 \%)$ \\
\hline Lack of transport & $59(17.7 \%)$ & 45 (19.7\%) & $17(24.6 \%)$ & $31(29.8 \%)$ & 0.049 & $152(20.7 \%)$ & $10(15.6 \%)$ \\
\hline
\end{tabular}

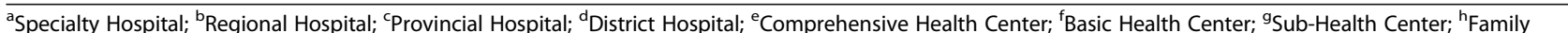
Health House 
and over $24 \%$ of respondents from low volume $\mathrm{DH} /$ $\mathrm{CHC}$ and $\mathrm{BHC} / \mathrm{SHC} / \mathrm{FHH}(p=0.049)$.

\section{Snapshot of private health facilities}

In 18 of $20(90 \%)$ private health facilities, facility management reported providing 24-h services. Sixteen of 64 SBAs (25\%) had received training in both BEmONC and $\mathrm{PE} / \mathrm{E}$ management in the last 3 years (Table 1 ).

Of the 20 private health facilities, 18 (90\%) had injectable MgSO4, 15 (75\%) had injectable calcium gluconate, $18(90 \%)$ had oxytocin and $12(60 \%)$ had misoprostol available in the delivery room. Fifteen $(75 \%)$ had functioning BP apparatus, 17 of $20(85 \%)$ had a functioning stethoscope, $15(75 \%)$ had a functioning fetoscope and 7 (35\%) had EmONC guidelines and protocols in the delivery room (Table 2).

Of 64 SBAs interviewed at private facilities, 54 (84.4\%) correctly identified a case with severe headache, blurred vision, BP $160 / 120$, and $3+$ proteinuria as severe pre-eclampsia. In addition, 56 of 64 (87.5\%) SBAs recognized administration of $\mathrm{MgSO} 4$ as an essential action for management of severe pre-eclampsia; 27 of 64 (42.2\%) SBAs knew to prepare pregnant women with severe pre-eclampsia for childbirth within $24 \mathrm{~h}$, and 34 of 64 (53.1\%) SBAs knew antihypertensive medication as essential action for management of severe pre-eclampsia (Table 3).

SBAs asked if women had recently or were currently experiencing severe headache or blurred vision in only 9 of 45 (20\%) ANC consultations, 7 of $24(29.2 \%)$ women observed during childbirth and 5 of 30 (16.7\%) women observed in the postnatal ward. In 3 of 45 (6.7\%) ANC and 6 of 30 (20\%) PNC consultations, SBAs counseled women on the need for care seeking if experiencing convulsions or loss of consciousness. Likewise, they provided counseling on the need for care seeking if experiencing severe headache with blurred vision in 3 of 45 (6.7\%) ANC consultations and 6 of 30 (20\%) PNC consultations (Table 4).

Of 64 SBAs interviewed at private facilities, 45 (70.3\%) reported that their supervisors treat them with respect and $47(73.4 \%)$ mentioned a need for more knowledge and training. Security was not a concern among most SBAs interviewed; only 10 of $64(15.6 \%)$ respondents stated good security in the workplace as an important factor in enabling environment for quality care service provision.

\section{Discussion}

Our study shows numerous gaps in knowledge and practices required for early detection and management of $\mathrm{PE} / \mathrm{E}$ at both public and private health facilities. Although most facilities had supplies and medicines required for early detection and management of $\mathrm{PE} / \mathrm{E}$, gaps were identified in availability of supplies, medicines and equipment in various health facilities.

All SBAs should know the essential actions in management of severe $\mathrm{PE} / \mathrm{E}$, which are administration of $\mathrm{MgSO} 4$, antihypertensive drugs, and timely childbirth [18]. Although most SBAs across all public and private health facilities correctly diagnosed PE/E based on case descriptions, almost half of them, however, did not recognize antihypertensive drug administration and timely delivery as essential actions. In contrast, the 2010 Afghanistan EmONC assessment findings showed that the majority of SBAs could describe essential management of $\mathrm{PE} / \mathrm{E}$ in case scenarios. In addition, trained SBAs had higher knowledge than untrained SBAs, and the majority of them had received EmONC training [9]. These findings demonstrate that SBA knowledge in this assessment might be lower than in the 2010 study. SBAs' knowledge should be improved through evidence-based capacity-building approaches such as low-dose, high-frequency (LDHF) training that proved effective in Ghana [19].

The majority of maternal deaths due to $\mathrm{PE} / \mathrm{E}$ could be averted with early detection and timely delivery before reaching severe PE/E and eclampsia [20]. Our study found that not all women had their BP and proteinuria checked when needed, or danger signs assessed. Studies have shown that gaps between knowledge and clinical performance exist in part because of low motivation and health system constraints and training and supervision without a focus on effectiveness are unlikely to result in the improvements needed to ensure quality of care [21]. To change SBAs' behavior, it may be important to focus on cognition, attitudes, and motivation of health care providers to improve quality of care [22].

Studies of PE/E case management in Nigeria and Pakistan identified similar gaps in quality of care. In Nigeria, use of $\mathrm{MgSO} 4$ was inconsistent in relation to standards [23]. In Pakistan, the majority of hospitals used $\mathrm{MgSO} 4$ as the preferred anticonvulsant to manage eclampsia, but there were large variations in dosages and in most cases doses were not in line with international guidelines [24]. In many settings, even when $\mathrm{MgSO} 4$ is available and providers know what drug to give, they struggle with its correct use.

Recent evidence supports multiple repetitive learning sessions that provide opportunities to practice skills and mechanisms for fostering interaction along with reinforcement of key messages as components of the learning support to improve quality of care [25].

In 2010, diazepam was used in almost half of the hospitals for management of PE/E [9]. Our findings, six years later, showed that the majority of SBAs recognized $\mathrm{MgSO} 4$ as the preferred anticonvulsant. This is consistent 
with findings from a study in Northern Afghanistan [26]. The positive shift from use of diazepam to $\mathrm{MgSO} 4$ over a period of time might be the result of a focused quality improvement program, which stimulated the translation of policies into practice in health facilities as well as distributing job aids and improving compliance with the national PE/E protocols [27].

Knowledge gaps demonstrated by more than half of SBAs in public health facilities and even more in private health facilities underscored the need for further investments in healthcare provider capacity building within quality improvement efforts.

Strategies found to be cost-effective in other settings include a combination of classroom learning and practice with simulators during and between repetitive sessions of peer-led practice and feedback. Follow-up via targeted Short Message Service (SMS) messaging and support by mentors will sustain the input [19, 28]. Recent evidence shows that a strategy for performance improvement of health care providers is more effective when training is combined with supervision and group based problem solving rather than training in isolation [29].

Policymakers and health managers need to review existing effective capacity-building approaches and develop a short-and long-term strategy for SBAs performance improvement across public and private health facilities. Ensuring good birth outcomes depend upon processes of care being performed correctly and consistently and complying with evidence based standards needs additional attention including monitoring progress [30].

Unavailability of essential medicines increases the risk of maternal and perinatal mortality and morbidity. The main reason for such shortages in Afghanistan could be supply chain management problems. Sharma et al. (2017) suggest that strengthening existing national logistics management systems to ensure adequate forecasting, supply, and availability of essential drugs and commodities through the establishment of web-based logistics management information systems could be a solution [31].

Many factors influence SBAs' performance on quality intrapartum and postnatal care, such as access to training and supervision, workload, salaries and living conditions, and access to well-equipped and well-organized health facilities and transport [32]. In addition to lack of training opportunities, we found that lack of respectful supervision was a challenge for more than half of SBAs across public and private health facilities. This finding is consistent with study results in other low-resource settings [31].

Security is a key factor affecting SBAs' performance in health facilities in Afghanistan. The security situation was stressed as an important factor affecting both the work environment and travel between home and the health facility in public health facilities. Since most private hospitals are located in the capitals of the provinces, there are fewer security concerns.

Although this study was not designed to provide a representative picture of private sector health facilities or directly compare readiness and quality of care in public versus private health facilities, a snapshot suggests that quality of care in early detection and management of PE/E might be similar.

\section{Limitations}

This assessment had several limitations. Data collection at low- and high-volume facilities was not conducted concurrently, so it might not be possible to generalize influences of seasonal conditions and insecurity on health services. The data collected on health services provided in the three months preceding the assessment is based on facility management or health care worker verbal reports and not documentation of services provided or triangulation of multiple data sources. Finally, because the study was designed to assess the quality of many aspects of $\mathrm{MNH}$ care, some aspects of $\mathrm{PE} / \mathrm{E}$ readiness, detection and case management practices, such as proteinuria and antihypertensive dosage for PE/E management, were not captured.

Although the data presented on private health facility readiness and service quality is not representative of all private facilities in Afghanistan and cannot be compared with nationally representative data from public health facilities, the anecdotal data presented may challenge assumptions that there are substantive differences in the quality of care provided at public and private sector facilities. There is a need for more rigorous and generalizable data on quality care provision at private facilities.

Despite these limitations, this study provides important evidence on gaps in quality of early detection and management of PE/E at public health facilities and some insight into the quality of care at private health facilities in Afghanistan.

\section{Conclusions}

While the unavailability of essential medicines at some health facilities increases the risk of maternal and perinatal mortality and morbidity in both public and private health facilities, the major challenge in early detection and management of PE/E across all levels of public and private health facilities in Afghanistan is to close the gaps in SBA knowledge and practice.

Appropriate and effective solutions are available to make a paradigm shift in how SBAs are supported at all health facility levels to deliver lifesaving services to 
mothers and newborns. Policymakers and health partners should review existing SBA capacity-building approaches and adopt evidence-based and cost-effective repetitive training approaches. Strengthening the supply management system at national and health facility levels through a web-based logistic information system is required to ensure that all facilities have essential medicines and supplies in place.

\section{Abbreviations}

ANC: Antenatal care; BEmONC: Basic emergency obstetric and newborn care; BHC: Basic health center; BP: Blood pressure; CHC: Comprehensive health center; DH: District hospital; EmONC: Emergency obstetric and newborn care; $\mathrm{FHH}$ : Family health house; LDHF: Low-dose, high-frequency; MCPC: Managing Complications in Pregnancy and Childbirth; MgSO4: Magnesium sulfate; MNH: Maternal and newborn health; MoPH: Ministry of public health; PE/E: Pre-eclampsia/eclampsia; PH: Provincia hospital; PNC: Postnatal care; RH: Regional hospital; SBA: Skilled birth attendant; SH: Specialized hospital; SHC: Sub-health center; WHO: World Health Organization

\begin{abstract}
Acknowledgements
The authors would like to thank UNICEF and USAID for funding the study and technical collaboration, central and provincial MoPHs for their guidance and support, the nongovernmental organizations implementing Afghanistan's health care services, and those professionals who participated in the study. Special thanks to Malalai Naziri, Ariel Higgins-Steele and Shirin Varkey from UNICEF; Sher Shah Amin from USAID; Sayed Attalla Saeedzai, MoPH General Director of Evaluation and Health Information System; Zelaikha Anwari, MoPH Director of Reproductive, Maternal, Newborn Child and Adolescent Health Department; Catherine Todd, Reproductive, Maternal, Newborn Child Health Division; and Pasty Bailey, Senior Scientist Health Services Division, FHI360; Faridullah Atiqzai, Zahra Sultani, Sayed Esmati, Ahmad Eklil Husain, Mahmood Azimi, Enayatullah Mayar, Abdul Qader Rahimi, Raouf Saidzadah, Matiuallah Noorzad, and Laila Natiq from Jhpiego Afghanistan; Elizabeth Sasser and Grace Jacoby from Jhpiego/Baltimore; facility staff participating in the study; and women allowing observation of care. Thanks to all the midwives and doctors who served as data collectors in very difficult situations.
\end{abstract}

\section{Funding}

The United States Agency for International Development (USAID) Afghanistan FP/MNCH Project (AID-306-A-15-00002) and UNICEF Afghanistan National Maternal and Newborn Health Quality of Care Assessment award provided funding for this research. The contents of this manuscript are the responsibility of the authors and do not necessarily reflect the views of the funder.

\section{Availability of data and materials}

Data is available from the Ministry of Public Health upon request. Requests should be directed to the MoPH's Evaluation and Health Information Systems Department (ehis.moph@gmail.com),

\section{Authors' contributions}

NA served as co-investigator for the 2016 Afghanistan National Maternal and Newborn Health Quality of Care Assessment, contributed to analysis and interpretation of study findings, and wrote the first draft of the manuscript. PM and SC served as co-investigators, and contributed to the interpretation of study findings, reviews, and revision of the manuscript. FM contributed to the analysis and interpretation of study findings and revision of the manuscript. JVR, JS, and YMK served as study advisory board members and contributed to the review and revision of the manuscript. HT served as principal investigator, led the data analysis, and contributed to the interpretation of study findings and revision of the manuscript. All authors read and approved the final manuscript.

\section{Ethics approval and consent to participate}

The 2016 National Maternal and Newborn Health Quality of Care Assessment protocol was approved by the ethical review boards of the Afghanistan MoPH (IRB\# 361533) and John Hopkins Bloomberg School of Public Health in Baltimore, Maryland (IRB\# 6799). Written permission for data collection was obtained from facility directors, and oral informed consent was obtained from all participating health care providers and women (or a woman's next of kin if they were too ill to provide informed consent themselves).

\section{Competing interests}

The authors declare that they have no competing interests.

\section{Publisher's Note}

Springer Nature remains neutral with regard to jurisdictional claims in published maps and institutional affiliations.

\section{Author details \\ ${ }^{1}$ Jhpiego, 1615 Thames Street, Baltimore, MD, USA. ${ }^{2}$ Athena Institute, Faculty of Science, Vrije Universiteit, Amsterdam, De Boelelaan 1105, 1081, HV, Amsterdam, the Netherlands. ${ }^{3}$ Jhpiego Afghanistan, Kabul, Afghanistan. ${ }^{4}$ Department of Health Sciences, Global Health, University of Groningen, University Medical Centre Groningen, Groningen, the Netherlands. ${ }^{5}$ Department of Obstetrics and Gynecology, Leeuwarden Medical Centre, Leeuwarden, the Netherlands.}

Received: 27 July 2018 Accepted: 11 December 2018

Published online: 18 January 2019

\section{References}

1. Say L, Chou D, Gemmill A, Tucalp Ö, Moller AB, Daniels J, et al. Global causes of maternal death: a WHO systematic analysis. Lancet Glob Health 2014;2:323-3.

2. Mol BWJ, Roberts CT, Thangaratinam S, Magee LA, De Groot CJM, Hofmeyr GJ. Pre-eclampsia. Lancet. 2016;387:999-1011.

3. Blencowe H, Cousens S, Chou D, Oestergaard M, Say L, Moller AB, et al. Born too soon : the global epidemiology of 15 million preterm births. Reprod Health. 2013;10(Suppl 1):1-13.

4. Kassebaum NJ, Barber RM, Bhutta ZA, Dandona L, Gething PW, Hay SI, et al. Global, regional, and national levels of maternal mortality, 1990-2015: a systematic analysis for the Global Burden of Disease Study 2015. Lancet. 2016:388(10053):1775-812.

5. Afghan Public Health Institute, Ministry of Public Health (APHI/MoPH) [Afghanistan], Central Statistics Organization (CSO) [Afghanistan], ICF Macro, Indian Institute of Health Management Research (IIHMR) [India], and World Health Organization Regional Office for the Eastern Mediterranean (WHO/ EMRO) [Egypt]. 2011. Afghanistan Mortality Survey 2010. Calverton, Maryland, USA: APHI/MoPH, CSO, ICF Macro, IIHMR, and WHO/EMRO.

6. Bartlett L, LeFevre A, Zimmerman L, Saeedzai SA, Torkamani S, Zabih W, et al. Progress and inequities in maternal mortality in Afghanistan (RAMOSII): a retrospective observational study. Lancet Glob Health. 2017;5:e545-55.

7. World Health Organization (WHO). Prevention and treatment of preeclampsia and eclampsia. Geneva: WHO: 2011.

8. World Health Organization (WHO). Standards for improving quality of maternal and newborn care in health facilities. WHO. 2016. http://www. who. int/iris/handle/10665/249155. Accessed 21 May 2018.

9. Kim YM, Ansari N, Kols A, Tappis H, Currie S, Zainullah $\mathrm{P}$, et al. Prevention and management of severe pre-eclampsia/eclampsia in Afghanistan. BMC Pregnancy Childbirth. 2013:13(1):186.

10. United Nations Assistance Mission in Afghanistan (UNAMA). Afghanistan: Protection of civilians in armed conflict. In: Annual report 2016. Kabul: UNAMA; 2017. https://unama.unmissions.org/sites/default/files/protection_ of_civilians_in_armed_conflict_annual_report_8feb_2016.pdf. Accessed 21 May $201 \overline{8}$

11. World Health Organization (WHO). Heatlh cluster bulletin. Health cluster Afghanistan. In: WHO; 2017.

12. Bartlett L, Cantor D, Lynam P, Kaur G, Rawlins B, Ricca J, et al. Facility-based active management of the third stage of labour: assessment of quality in six countries in sub-Saharan Africa. Bull World Health Organ. 2015;93(11):759-67.

13. ICF. The Demographic Health Survey: Service Provsion Assessment (SPA) 2012. [https://dhsprogram.com/What-We-Do/Survey-Types/SPA.cfm] 
14. Averting Maternal Death and Disability Program (AMDD). Needs Assessment of Emergency Obstetric and Newborn Care (EmONC): Facilitator's Guide. New York: AMDD, Columbia University; 2009.

15. Ministry of Public Health (MoPH). The essential package of hospital services for Afghanistan. Kabul: Islamic Republic of Afghanistan MoPH; 2005. http:// moph.gov.af/Content/Media/Documents/EPHS-2005FINAL29122010164126629.pdf. Accessed 21 May 2018.

16. Ministry of Public Health (MoPH). A basic package of health services for Afghanistan 2010/1389. Kabul: Islamic Republic of Afghanistan MoPH; 2010.

17. Ministry of Public Health (MoPH). National Reproductive, maternal, newborn, child and adolescent health Strategy 2017-2021. Kabul: Islamic Republic of Afghanistan MoPH; 2017.

18. World Health Organization (WHO). Managing complications in pregnancy and childbirth: a guide for midwives and doctors. Geneva: WHO; 2007.

19. Gomez PP, Nelson AR, Asiedu A, Addo E, Agbodza D, Allen C, et al. Accelerating newborn survival in Ghana through a low-dose, highfrequency health worker training approach: a cluster randomized trial. BMC Pregnancy Childbirth. 2018;18(1):72.

20. Goldenberg RL, McClure EM, MacGuire ER, Kamath BD, Jobe AH. Lessons for low-income regions following the reduction in hypertension-related maternal mortality in high-income countries. Int J Gynecol Obstet. 2011:113:91-5

21. Leslie HH, Gage A, Nsona H, Hirschhorn LR, Kruk ME. Training and supervision did not meaningfully improve quality of care for pregnant women or sick children in sub-Saharan Africa. Health Aff 2016; 35(9):1716-1724, 1-19.

22. Grol R, Wensing M. What drives change? Barriers to and incentives for achieving evidence-based practice. Med J Aust. 2004;180(6 Suppl):57-60.

23. Danmusa S, Coeytaux F, Potts J, Wells E. Scale-up of magnesium sulfate for treatment of pre-eclampsia and eclampsia in Nigeria. Int I Gynecol Obstet. 2016;134:233-6

24. Bigdeli M, Zafar S, Assad H, Ghaffar A. Health system barriers to access and use of magnesium sulfate for women with severe Pre-eclampsia and eclampsia in Pakistan: evidence for policy and practice. PLoS One. 2013;8(3):1-9.

25. Bluestone J, Johnson P, Fullerton J, Carr C, Alderman J, BonTempo J. Effective in-service training design and delivery: evidence from an integrative literature review. Hum Resour Health. 2013;11(51).

26. Hamajima N, Reyer JA, Rabi M, Yoshida Y, Sarker MAB, Ahadi SSM. Clinical features, current treatments and outcome of pregnant women with preeclampsaia/eclampsia in northern Afghanistan. Nagoya J Med Sci. 2015;77(1-2):103-11.

27. Rahimzai M, Amiri M, Burhani NH, Leatherman S, Hiltebeitel S, Rahmanzai AJ. Afghanistan's national strategy for improving quality in health care Int J Qual Health Care. 2013;25:270-6.

28. Willcox M, Harrison H, Asiedu A, Nelson A, Gomez P, Lefevre A. Incremental cost and cost-effectiveness of low-dose, high-frequency training in basic emergency obstetric and newborn care as compared to status quo: part of a cluster-randomized training intervention evaluation in Ghana. Glob Health. 2017;13:88.

29. Rowe AK, Rowe SY, Peters DH, Holloway KA, Chalker J, Ross-Degnan D. Effectiveness of strategies to improve health-care provider practices in lowincome and middle-income countries: a systematic review. Lancet Glob Heal. 2018;6:e1163-75.

30. World Health Organization (WHO). Standards for improving jQuality of Maternal and Newborn Care in Health Facilities. Geneva, WHO; 2016.

31. Sharma G, Mathai M, Dickson KE, Weeks A, Hofmeyr GJ, Lavender T, et al. Quality care during labour and birth : a multi-country analysis of health system bottlenecks and potential solutions. BMC Pregnancy Childbirth. 2015;15(Suppl 2):S2.

32. Munabi-Babigumira S, Glenton C, Lewin S, Fretheim A, Nabudere H. Factors that influence the provision of intrapartum and postnatal care by skilled birth attendants in low- and middle-income countries: a qualitative evidence synthesis. Cochrane Database Syst Rev. 2015. https://doi.org/10. 1002/14651858.CD011558.

Ready to submit your research? Choose BMC and benefit from:

- fast, convenient online submission

- thorough peer review by experienced researchers in your field

- rapid publication on acceptance

- support for research data, including large and complex data types

- gold Open Access which fosters wider collaboration and increased citations

- maximum visibility for your research: over $100 \mathrm{M}$ website views per year

At BMC, research is always in progress.

Learn more biomedcentral.com/submissions 